\title{
Abordagem da disfunção tiroideia subclínica
}

Natália Guerreiro*

\section{RESUMO}

Objectivos: A disfunção tiroideia subclínica é um problema prevalente. No entanto, existe, ainda, controvérsia quanto à sua abordagem. É objectivo da presente revisão sistematizar essa abordagem com base na evidência científica actual.

Metodologia: Foi realizada uma pesquisa de artigos publicados nas bases de dados Medline/Pubmed, Cochrane Library, Medscape, entre 1998 e 2008. Dos 226 artigos encontrados, atendendo ao interesse clínico e tipo de estudo, foram seleccionados 15.

Resultados: A disfunção tiroideia subclínica inclui o hipotiroidismo subclínico e o hipertiroidismo subclínico. A progressão para hipotiroidismo clínico ocorre em $2 \%$ a $5 \%$ por ano, sendo mais provável com valores de Hormona Estimulante da Tiróide (TSH) elevados e presença de anticorpos anti-peroxidase. É recomendado iniciar tratamento com levotiroxina quando o nível de TSH é superior a $10 \mathrm{mU} / \mathrm{l}$. Quando o nível de TSH se encontra entre 4,5 a $10 \mathrm{mU} / \mathrm{l}$ devem tratar-se os doentes sintomáticos e as grávidas. A progressão para hipertiroidismo clínico pode chegar aos $5 \%$ por ano, sendo o risco de progressão maior se existir doença tiroideia prévia ou consumo de amiodarona. Os casos confirmados de hipertiroidismo subclínico devem ser referenciados a um serviço de Endocrinologia, para se efectuar o diagnóstico etiológico e optimização da terapêutica. A monitorização do doente deve ter uma periodicidade adaptada à fase do tratamento.

Conclusão: Não há evidência clara das consequências clínicas da disfunção tiroideia subclínica não tratada e dos benefícios do seu tratamento. Até que estudos de larga escala estejam disponíveis, a melhor abordagem consiste no julgamento clínico, tendo em conta a sintomatologia, os níveis séricos de TSH e a preferência do doente.

Palavras-chave: Abordagem; Disfunção Tiroideia Subclínica.

\section{INTRODUÇÃO}

A disfunção tiroideia subclínica é uma situação com elevada prevalência. $\mathrm{O}$ hipotiroidismo subclínico tem uma prevalência de 4 a $10 \%$ na população geral, ${ }^{1-7}$ e de $20 \%$ nas mulheres com idade superior a 60 anos. ${ }^{1,2,6} \mathrm{~A}$ prevalência do hipertiroidismo subclínico é menor e mais variável em função dos estudos: $0,7 \%$ (estudo de Joseph), ${ }^{8} 2 \%$ (estudo Colorado), ${ }^{1,2}$ e $12 \%$ (estudo de Framingham). ${ }^{8}$

Apesar de se tratar de um problema comum, que surge com frequência em Cuidados de Saúde Primários e de poder ter consequências clínicas importantes existe, ainda, controvérsia quanto à sua abordagem e orientação.

O objectivo desta revisão é sistematizar a abordagem da disfunção tiroideia subclínica, com base na evidência científica actual. Focam-se os seguintes aspectos: definição, consequências clínicas, factores de ris-

*Interna de Medicina Geral e Familiar

USF BRIOSA - Centro de Saúde Norton de Matos (Coimbra) co de progressão para doença clínica, indicações para terapêutica e sua monitorização.

\section{MÉTODOS}

Foi efectuada uma pesquisa sistemática nas bases de dados Medline/Pubmed, Medscape, Cochrane Library, utilizando as palavras-chave «subclinical thyroid disease». Foram incluídos, apenas, artigos publicados entre 1998 e 2008, de acesso gratuito, em espanhol, inglês e português. Dos 226 artigos encontrados, atendendo ao interesse clínico e tipo de estudo, foram seleccionados 15 , dos quais quatro estudos controlados aleatorizados, nove revisões sistemáticas e duas normas de orientação clínica.

\section{HIPOTIROIDISMO SUBCLÍNICO}

Diagnóstico

O diagnóstico do hipotiroidismo subclínico é laboratorial, sendo considerado quando a concentração sérica de Hormona Estimulante da Tiróide (TSH) se encontra acima da definição estatística considerada como o 
limite superior do normal (geralmente acima de $4,5 \mathrm{mU} / \mathrm{l}$ ) e o valor de tiroxina livre (T4 livre) está dentro dos valores de referência, que podem variar de acordo com os diferentes laboratórios. ${ }^{1,2,7}$ A sensibilidade funcional deve ser estabelecida por cada laboratório e o método usado para avaliar a TSH deve ter uma sensibilidade funcional de, pelo menos, $0,02 \mathrm{mU} / \mathrm{l}^{2}{ }^{2}$

Existe muita controvérsia acerca da realização do rastreio da doença tiroideia em doentes assintomáticos. De acordo com a American Thyroid Association, devem ser rastreados homens e mulheres com mais de 35 anos, com uma periodicidade de 5 anos. ${ }^{15}$ A American Association of Clinnical Endocrinologists considera o rastreio nos idosos, particularmente mulheres..$^{15} \mathrm{O}$ American College of Physicians recomenda-o nas mulheres com mais de 50 anos e com um ou mais sintomas que possam ser atribuídos a patologia tiroideia. ${ }^{15}$ A United States Preventive Services Task Force considera não existir evidência suficiente para recomendar ou não o rastreio, e a Royal College of Physicians considera o rastreio não justificado. ${ }^{15}$ Segundo a British Thyroid Association o rastreio na população geral saudável não é necessário, mas pode ser realizado em mulheres pós-menopáusicas ou com sintomas inespecíficos (nível de evidência IV, grau de recomendação C) (Quadro I). ${ }^{7}$

A maioria dos autores considera que, a realizar-se o rastreio, este seria através do doseamento da TSH. ${ }^{15}$

A avaliação da função tiroideia, de acordo com a British Thyroid Association, deve ser realizada às grávidas ou, preferencialmente, às mulheres que pretendem en-

$\begin{aligned} & \text { QUADRO I. Grau de Recomendação, adaptado da } \\ & \text { Agency for Health Care Policy and Research. }\end{aligned}$
Grau

gravidar, que apresentem um dos seguintes problemas: diabetes tipo 1, patologia tiroideia prévia ou actual, história familiar de doença tiroideia, bócio ou sintomas de hipotiroidismo (nível de evidência IIb, grau de recomendação B). Os testes de função tiroideia durante a gravidez devem ser a TSH e T4 livre (nível de evidência II, grau de recomendação B). O doseamento dos anticorpos anti-peroxidase (anti-TPO) deve também ser considerado, uma vez que tem valor preditivo para a tiroidite do pós-parto e atingimento fetal (nível de evidência IV, grau de recomendação C).?

\section{Consequências clínicas}

Foram identificadas várias consequências clínicas do hipotiroidismo subclínico, algumas das quais controversas. De acordo com uma revisão sistemática de grandes dimensões, há evidência suficiente da associação do hipotiroidismo subclínico com dislipidemia, apenas quando o valor de TSH é superior a 10 mU/l. Não existe evidência suficiente que comprove a associação a aterosclerose, disfunção cardíaca, sintomas de hipotiroidismo sistémico ou neuropsiquiátricos. Estes últimos dados resultam de estudos de menores dimensões, daí a associação não ser estatisticamente significativa. (Quadro II e III). ${ }^{2}$

\section{Factores de risco de progressão para doença clínica} A existência de valores elevados de TSH aumenta a pro-

\begin{tabular}{|c|c|c|}
\hline \multicolumn{3}{|c|}{$\begin{array}{l}\text { QUADRO II. Qualidade de evidência na força de } \\
\text { associação do hipotiroidismo subclínico e } \\
\text { consequências clínicas. }{ }^{2}\end{array}$} \\
\hline \multirow[b]{2}{*}{ Consequência clínica } & \multicolumn{2}{|c|}{$\begin{array}{c}\text { Força de associação } \\
\text { Qualidade de evidência }\end{array}$} \\
\hline & $\begin{array}{c}\text { TSH 4,5 a } \\
10 \mathrm{mU} / \mathrm{l}\end{array}$ & $\begin{array}{c}\text { TSH } \\
>10 \mathrm{mU} / \mathrm{l} \\
\end{array}$ \\
\hline $\begin{array}{l}\text { Progressão para } \\
\text { hipotiroidismo }\end{array}$ & Boa & Boa \\
\hline Aterosclerose & Insuficiente & Insuficiente \\
\hline $\begin{array}{l}\text { Aumento do colesterol } \\
\text { total e LDL }\end{array}$ & Insuficiente & Suficiente \\
\hline Disfunção cardíaca & $*$ & Insuficiente \\
\hline Sintomas de hipotiroidismo & Não existe & Insuficiente \\
\hline Sintomas neuropsiquiáticos & Não existe & Insuficiente \\
\hline
\end{tabular}

*os estudos não fizeram distinção entre os valores de TSH 
QUADRO III. Qualidade de evidência, adaptada de US Preventive Services Task Force. ${ }^{2}$

\section{Boa}

A evidência inclui resultados consistentes de estudos bem desenhados, bem controlados em populações representativas Suficiente

A evidência é suficiente para determinar efeitos na saúde, mas a força de evidência é limitada pelo número, qualidade ou consistência dos estudos individuais, ou generalização à prática clínica

\section{Insuficiente}

A evidência é insuficiente pelo número limitado ou qualidade dos estudos, falhas importantes no seu desenho ou aplicação, ou falta de informação acerca dos efeitos na saúde

babilidade de progressão do hipotiroidismo subclínico para hipotiroidismo clínico, sendo esta proporcional ao nível sérico de TSH.,8-11 Se os valores de TSH forem superiores a $10 \mathrm{mU} / \mathrm{l}$, a probabilidade de progressão é de $5 \%$ por ano, se forem superiores a $14 \mathrm{mU} / \mathrm{l}$ a probabilidade é de $20 \%$ por ano, e se forem superiores a 20 $\mathrm{mU} / \mathrm{l}$, em doentes com mais de 65 anos, a progressão é de $80 \%$ por ano. ${ }^{2}$

Admite-se que a presença de anticorpos anti-TPO confere, praticamente, o dobro de probabilidade de progressão ( $4,3 \%$ por ano, se presentes, contra $2,6 \%$ por ano, se ausentes). ${ }^{2,8,10-13}$ No entanto, há controvérsia acerca da necessidade do seu doseamento por rotina. O Consenso de 2002 da American Association of Clinical Endocrinologists (AACE), American Thyroid Association (ATA) e The Endocrine Society (TES) considera que não existe evidência para recomendar a favor, ou contra, o doseamento de rotina destas anticorpos. ${ }^{1}$ Pelo contrário, o Royal College of Physicians recomenda a determinação dos anticorpos anti-TPO em todos os doentes com disfunção tiroideia subclínica. Esta instituição fundamenta a sua recomendação no facto de os anti-TPO serem um factor a ter em conta na decisão de iniciar a terapêutica, predizerem o risco de progressão da doença e assinalarem um risco aumentado de outras doenças auto-imunes, como a Diabetes Mellitus tipo 1 ou a insuficiência suprarenal. ${ }^{6} \mathrm{~A}$ British Thyroid Association também recomenda o do- seamento de anticorpos anti-TPO (nível de evidência III, grau de recomendação B). ${ }^{7}$ Os anticorpos anti-tireoglobulina (anti-TG) não têm valor preditivo de progressão. ${ }^{8}$

Foram, também, identificados, como factores de risco que aumentam a probabilidade de progressão para doença clínica, os seguintes: antecedentes de hipertiroidismo, ${ }^{2,11}$ Diabetes Mellitus tipo $1,{ }^{2}$ terapêutica com lítio, ${ }^{11}$ radioterapia da cabeça e pescoço, ${ }^{2,11}$ antecedentes familiares de patologia tiroideia. ${ }^{2}$

\section{Indicações para tratamento}

Há, ainda, alguma controvérsia acerca dos factores que devem ser tidos em conta para iniciar o tratamento com levotiroxina nos doentes com hipotiroidismo subclínico. De acordo com a British Thyroid Association, 2006, é indicação para iniciar tratamento a existência de níveis de TSH superiores a $10 \mathrm{mU} / \mathrm{l}$ (nível de evidência II, grau de recomendação B). Se os valores de TSH estiverem entre 4,5 a $10 \mathrm{mU} / \mathrm{l}$ não se recomenda o tratamento por rotina (nível de evidência II, grau de recomendação B), devendo, no entanto, ponderar-se, se o doente tiver bócio, for uma mulher grávida ou pretender engravidar. ${ }^{7}$ Estas recomendações baseiam-se na evidência de que, para valores de TSH moderadamente elevados (média 11,7 mU/l), o tratamento melhora o perfil lipídico e reduz os sintomas (nível de evidência Ib). ${ }^{7}$ O Consenso da AACE, ATA e TES, de 2002, recomenda, como indicações para início do tratamento, valores de TSH superiores a $10 \mathrm{mU} / \mathrm{l}$. Se o valor de TSH estiver entre 4,5 a $10 \mathrm{mU} / \mathrm{l}$, ponderar o tratamento apenas se o doente estiver sintomático. ${ }^{1}$

Alguns autores consideram, ainda, outros factores na decisão de iniciar o tratamento: tabagismo, ${ }^{14}$ dislipidemia, ${ }^{15}$ infertilidade, ${ }^{15}$ disfunção cardíaca ${ }^{15}$ e antecedentes familiares de patologia tiroideia. ${ }^{14}$ No entanto, existe menos consenso a este respeito.

Está recomendado iniciar o tratamento com levotiroxina com doses de 50 a $100 \mathrm{mcg}$ por dia, com ajustes de 25 a 50 mcg (nível de evidência IIb).Se o doente for idoso ou sofrer de cardiopatia isquémica, deve iniciar-se com doses mais baixas: $25 \mathrm{mcg}$, com ajustes de 25 mcg. ${ }^{7}$ A maioria dos doentes consegue a normalização da TSH com doses de 75 a $150 \mathrm{mcg}$ por dia $(1,6 \mathrm{mcg} /$ $/ \mathrm{Kg})^{7}$

Tendo em conta os estudos referidos, os níveis de 


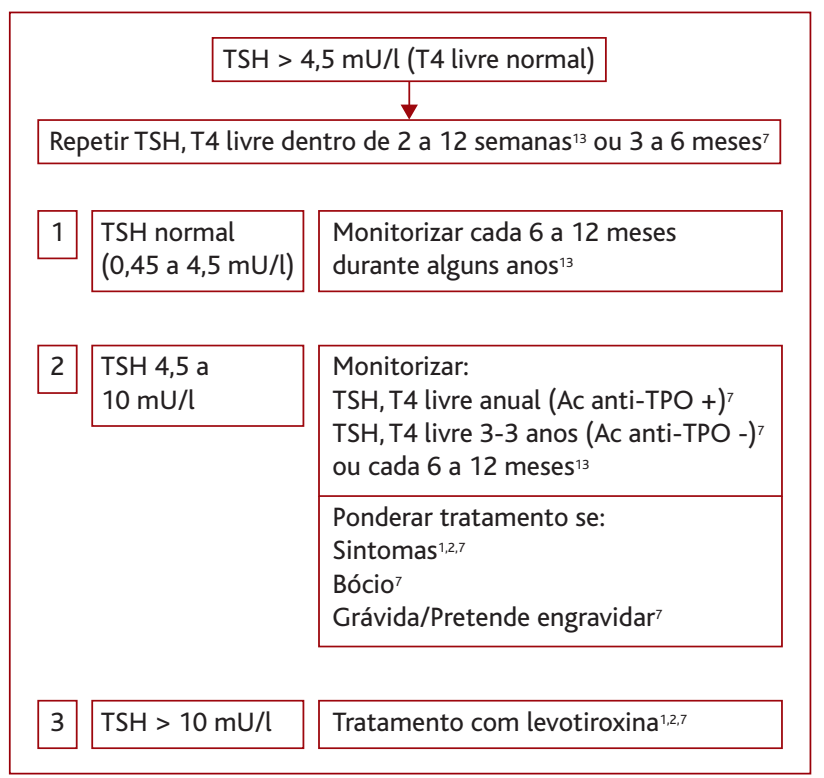

Figura 1. Algoritmo da abordagem do hipotiroidismo subclínico em Cuidados de Saúde Primários.

evidência e reflectindo as recomendações neles contidas, propõe-se um algoritmo de abordagem do hipotiroidismo subclínico em Cuidados de Saúde Primários (Figura 1).

\section{Monitorização do tratamento}

A monitorização da terapêutica implica o doseamento de TSH e T4 livre dois a três meses após o ajuste da dose, durante a optimização do tratamento (nível de evidência IV, grau de recomendação C); depois de alcançada a dose estável de levotiroxina, é suficiente o doseamento anual de TSH, para averiguar a compliance do doente e a necessidade de ajustes na dosagem (nível de evidência III, grau de recomendação B). ${ }^{7}$ Se o doente não tiver indicação para tratamento com levotiroxina e os anticorpos anti-TPO forem positivos, a monitorização recomendada é o doseamento anual de TSH e T4 livre; se os anticorpos forem negativos, o doseamento poderá ser efectuado de 3 em 3 anos (nível de evidência IV, grau de recomendação C). ${ }^{7}$

\section{HIPERTIROIDISMO SUBCLÍNICO \\ Diagnóstico}

O diagnóstico do hipertiroidismo subclínico estabelece-se quando o valor de TSH é inferior a 0,45 $\mathrm{mU} / \mathrm{l}$ e os valores de T4 livre e triiodotironina livre (T3 livre) se encontram dentro dos valores de referência (nível de evidência II, grau de recomendação B). ${ }^{7}$ É necessário um doseamento de T3 livre normal, uma vez que existe uma forma de tirotoxicose dependente de T3 livre. ${ }^{?}$

\section{Consequências clínicas}

Há boa evidência da associação do hipertiroidismo subclínico com a fibrilhação auricular, e existe evidência suficiente da associação com a disfunção cardíaca e diminuição da densidade mineral óssea. Estas associações verificam-se, apenas, quando o valor de TSH é inferior a $0,1 \mathrm{mU} / \mathrm{l}$ (Quadro III e IV). ${ }^{2}$

\section{Factores de risco de progressão para doença clínica}

Estão identificados, como factores que condicionam um aumento do risco de progressão do hipertiroidismo subclínico para hipertiroidismo clínico, os seguintes: antecedentes de doença tiroideia prévia, ${ }^{2}$ bócio, ${ }^{2}$ fibrilhação auricular ${ }^{2}$ e terapêutica com amiodarona. ${ }^{2} \mathrm{~A}$ progressão varia de $1 \%^{2}$ a $5 \%^{8}$ por ano.

\section{Indicações para tratamento}

A British Thyroid Association, 2006, recomenda a confirmação do hipertiroidismo subclínico através do doseamento de TSH, T3 e T4 livres, 3 a 6 meses depois do doseamento inicial (se o doente não sofrer de doença cardiovascular). Recomenda ainda a referenciação aos Cuidados de Saúde Secundários, para a realização do diagnóstico etiológico e optimização da terapêutica (nível de evidência III, grau de recomendação B). ${ }^{7} \mathrm{O}$ Consenso da AACE, ATA e TES, de 2002, recomenda o tratamento com fármacos antitiroideus se o valor de TSH for inferior a $0,1 \mathrm{mU} / \mathrm{l}^{1}$

Também aqui se propõe um algoritmo de abordagem do hipertiroidismo subclínico em Cuidados de Saúde Primários, que pretende ser o reflexo da análise efectuada (Figura 2).

\section{Monitorização do tratamento}

Está recomendado o doseamento da TSH, T3 livre e T4 livre, 1 a 3 meses após cada ajuste da dose dos fármacos antitiroideus, durante a optimização da terapêutica (nível de evidência III, grau de recomendação B). Depois da estabilização preconiza-se o doseamento anual (nível de evidência III, grau de recomendação B). ${ }^{7}$ 


\section{QUADRO IV. Qualidade de evidência na força de associação do hipertiroidismo subclínico e consequências clínicas. ${ }^{2}$}

\begin{tabular}{l|l|l}
\multirow{4}{*}{ Consequência clínica } & \multicolumn{2}{|c}{$\begin{array}{c}\text { Força de associação - } \\
\text { Qualidade de evidência }\end{array}$} \\
\cline { 2 - 3 } $\begin{array}{l}\text { TSH 0,1 a } \\
\text { Progressão para }\end{array}$ & \multicolumn{1}{c}{ TSH } \\
hipertiroidismo & Insuficiente & Boa \\
\hline Fibrilhação auricular & Insuficiente & Boa \\
\hline Disfunção cardíaca & Insuficiente & Suficiente \\
\hline $\begin{array}{l}\text { Sintomas hipertiroidismo, } \\
\text { neuropsiquiátricos }\end{array}$ & Insuficiente & Insuficiente \\
\hline $\begin{array}{l}\text { Diminuição da densidade } \\
\text { mineral óssea }\end{array}$ & Não existe & Suficiente \\
\hline Fracturas ósseas & Não existe & Insuficiente \\
\hline
\end{tabular}

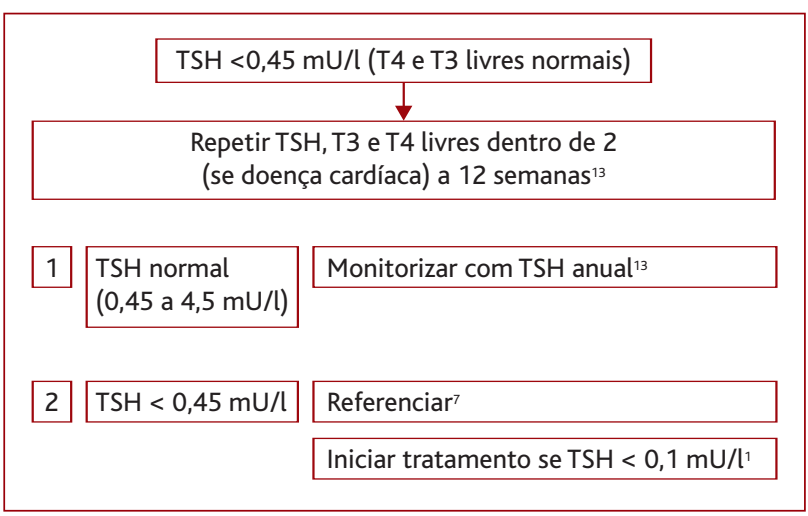

Figura 2. Algoritmo da abordagem do hipertiroidismo subclínico em Cuidados de Saúde Primários.

Se o doente não tiver indicação para tratamento farmacológico, deve monitorizar-se através do doseamento da função tiroideia, a cada 6 a 12 meses (nível de evidência III, grau de recomendação B). ${ }^{7}$

\section{CONCLUSÕES}

Existem dúvidas acerca das consequências da disfunção tiroideia subclínica e dos benefícios do tratamento, assim como da necessidade de rastrear a patologia tiroideia na população assintomática. Actualmente, de acordo com a evidência disponível, na decisão de iniciar o tratamento devem ter-se em conta os seguintes aspectos: presença de sintomatologia atribuível a pa- tologia tiroideia, valores de TSH, e a própria opinião do doente. Constitui indicação para o tratamento com levotiroxina a existência de valores de TSH superiores a $10 \mathrm{mU} / \mathrm{l}$. A terapêutica com fármacos antitiroideus está recomendada se os níveis de TSH forem inferiores a 0,1 mU/l. Dispondo de todos estes elementos, é possível tomar uma decisão mais adequada acerca do início do tratamento farmacológico da disfunção tiroideia subclínica.

São necessários mais estudos controlados aleatorizados, que avaliem as consequências clínicas da patologia tiroideia subclínica, bem como o benefício do início do tratamento.

\section{REFERÊNCIAS BIBLIOGRÁFICAS}

1. Gharib H, Tuttle M, Baskin J, Fish LH, Singer PA, McDermott MT. Consensus statement: Subclinical thyroid dysfunction: a joint statement on management from the American Association of Clinical Endocrinologists, the American Thyroid Association, and the Endocrine Society. J Clin Endocrinol Metab 2005 Jan; 90 (1): 581-5.

2. Surks MI, Ortiz E, Daniels GH, Sawin CT, Col NF, Cobin RH, et al. Subclinical thyroid disease: scientific review and guidelines for diagnosis and management. JAMA 2004 Jan; 291(2): 228-38.

3. Romaldini J, Sgarbi J, Farah C. Disfunções mínimas da tiróide: hipotiroidismo subclínico e hipertiroidismo subclínico. Arq Bras Endocrinol Metabol 2004 fev; 48 (1): 147-58.

4. Cooper DS. Subclinical thyroid disease: consensus or conundrum? Clin Endocrinol (Oxf) 2004 Apr; 60 (4): 410-2.

5. Rodondi N, Newman AB, Vittinghoff E, de Rekeneire N, Satterfield S, Harris TB, et al. Subclinical hypothyroidism and the risk of heart failure, other cardiovascular events, and death. Arch Intern Med 2005 Nov 28; 165 (21): 2460-6.

6. Takashima N, Niwa Y, Mannami T, Tomoike H, Iwan N. Characterization of subclinical thyroid dysfuncion from cardiovascular and metabolic viewpoints. Circ J 2007 Feb; 71 (2): 191-5.

7. Association for Clinical biochemistry, British Thyroid Association, British Thyroid Foundation. UK Guidelines for the Use of Thyroid Function Tests; 2006. Disponível em: www.acb.org.uk/docs/TFTguidelinefinal.pdf [acedido em 13/02/2009].

8. Kek P, Ho S, Khoo D. Subclinical thyroid disease. Singapore Med J 2003 Nov; 44 (11): 595-600.

9. Imaizumi M, Akahoshi M, Ichimaru S, Nakashima E, Hida A, Soda M, et al. Risk for ischemic heart disease and all-cause mortality in subclinical hypothyroidism. J Clin Endocrinol Metab 2004 Jul; 89 (7): 3365-70 .

10. Diez JJ, Iglesias P. Spontaneous subclinical hypothyroidism in patients older than 55 years: an analysis of natural course and risk factors for the development of overt thyroid failure.J Clin Endocrinol Metab 2004; 89: 4890-7.

11. Mcdermott M.,Ridgway C. Subclinical hypothyroidism is mild thyroid failure and should be treated. J Clin Endocrinol Metab 2001 Oct; 86 (10): 4585-90. 
12. Yeo CP, Gillett M, Vasikaran S. Suboptimal management of subclinical hypothyroidism. Med J Aust 2004 Aug 16; 181 (4): 232.

13. Col NF, Surks MI, Daniels GH. Subclinical thyroid disease: clinical applications. JAMA 2004 Jan 14; 291 (2): 239-43.

14. Chu JW, Crapo LM. The treatment of subclinical hypothyroidism is seldom necessary. J Clin Endocrinol Metab 2001 Oct; 86 (10): 4591-9.

15. Guha B, Krishnaswamy G, Peiris A. The diagnosis and management of hypothyroidism. South Med J 2002 May; 95 (5): 475-80.

\author{
ENDEREÇO PARA CORRESPONDÊNCIA \\ Natália Guerreiro Ferreira \\ Avenida Mendes Silva, $n^{\circ} 55,2^{\circ} \mathrm{C}$ \\ 3030-193 Coimbra \\ Tlm: 968892013 \\ E-mail: nataliaguerreiro@gmail.com \\ Recebido em 15/10/2008 \\ Aceite para publicação em 28/01/2009
}

\section{ABSTRACT}

Objectives: Subclinical thyroid dysfunction is a prevalent problem. Nonetheless, there are doubts about the best approach. The goal of this revision is systematize this approach based on actual scientific evidence.

Methods: A research was made from articles published in Pubmed/Medline, Cochrane Library and Medscape, between 1998 and 2008. From the 226 articles found, 15 were selected, based in clinical interest and type of study.

Results: Subclinical thyroid dysfunction includes subclinical hypothyroidism and subclinical hyperthyroidism. The progression to clinical hypothyroidism occurs in $2 \%$ to $5 \%$ a year. It is more likely when the value of thyroid stimulating hormone (TSH) is elevated and the antiperoxidase antibodies are present. Treatment with thyroxine should be initiated if the level of TSH is superior to $10 \mathrm{mU} / \mathrm{l}$. When the level of TSH is between $4,5 \mathrm{mU} / \mathrm{l}$ and $10 \mathrm{mU} / \mathrm{l}$, the treatment should be initiated only in symptomatic or pregnant patients. The progression to clinical hyperthyroidism can be $5 \%$ a year and is more likely if there is previous thyroid disease or ongoing treatment with amiodarone. The confirmed cases of hyperthyroidism should be sent to an endocrinologist service to determine the aetiology and optimize treatment. The monitorization should be adapted to treatment phase.

Conclusions: There is no clear evidence of clinical consequences of non treated subclinical thyroid dysfunction nor benefits of its treatment. Until further studies are available, the best approach should be based on clinical judgement, TSH levels and patients opinion.

Keywords: Approach; Subclinical Thyroid Dysfunction. 